\title{
Reconstructive Outcomes of Extensive Soft-Tissue Defects after Palliative Mastectomy for Advanced Breast Cancer
}

\author{
Taekeun Yoon (D), Soo Jin Woo (D), Ung Sik Jin (D) \\ Department of Plastic and Reconstructive Surgery, Seoul National University Hospital, Seoul National University College of Medicine, Seoul, Korea
}

\begin{abstract}
Background: In advanced breast cancer, reconstruction can be performed depending on the patient's situation, and can improve the subsequent treatment and quality of life. We examined the effect of reconstruction after palliative mastectomy on survival and quality of life. Methods: Between April 2010 and April 2021, 40 patients underwent reconstruction after palliative mastectomy for stage-IV breast cancer. To evaluate postoperative satisfaction, changes in the global health and quality-of-life scores after reconstruction were evaluated using questionnaires by the European Organisation for Research and Treatment of Cancer. The survival rate according to reconstructive surgery was estimated by the Kaplan-Meier survival analysis.

Results: Reconstruction methods included free transverse rectus abdominis musculocutaneous (TRAM) flap $(n=3)$, pedicled latissimus dorsi (LD) flap $(n=3)$, pedicled TRAM flap $(n=2)$, pedicled LD with pedicled vertical rectus abdominis musculocutaneous flap $(n=1)$, tissue expander insertion $(n=3)$, and split-thickness skin graft $(n=28)$. There were no major complications or decreased life expectancy due to reconstruction. All patients healed well and started conventional chemoradiotherapy at 31.3 days postoperatively. The quality-of-life scores increased from 37 to 83 after reconstruction. There was no difference in the survival rates between those who underwent reconstruction with split-thickness skin graft and flap operation $(P>0.05)$. The mean survival time postoperatively was 43.9 months.

Conclusion: Compared to primary closure, reconstruction of extensive soft-tissue defects after palliative mastectomy did not affect life expectancy or delay postoperative chemoradiotherapy; it led to an improved quality of life. Therefore, the extensive defects expected after palliative mastectomy should not influence indications for reconstruction surgery.
\end{abstract}

Keywords: Breast neoplasms; Mastectomy; Palliative care; Reconstructive surgical procedures; Survival analysis

\section{Introduction}

Palliative mastectomy is performed in patients diagnosed with advanced-stage breast cancer for symptomatic and local control [1]. The median survival of these patients is approximately 27.1 months [2]. The purpose of palliative mastectomy is to relieve the patient's symptoms, optimize local control, improve the overall health and quality of life, and even increase the life expectancy [3]. In cases where the tumor is not large, it is usually possible to perform primary closure after resection, and additional reconstruction is not required. However, if the tumor is large and extensive soft-tissue defects occur after resection, reconstruction is required [2].

There have been few studies on reconstruction after palliative mastectomy in patients with stage-IV breast cancer. In previous studies, reconstruction after mastectomy for palliative purposes was considered from two perspectives. The first was about which method was more effective when reconstructive surgery was required after palliative mastectomy [4]; the other was focused on whether cosmetic reconstruction
Original Article

Received: September 7, 2021

Revised: October 7, 2021

Accepted: October 14, 2021

Corresponding author: Ung Sik Jin, M.D., Ph.D.

Department of Plastic and Reconstructive Surgery, Seoul National University Hospital, Seoul National University College of Medicine, 101 Daehak-ro, Jongno-gu, Seoul 03080, Korea

Tel: +82-2-2072-2375

Fax: +82-2-741-3985

E-mail: usj1011@snu.ac.kr

This is an Open Access article distributed under the terms of the Creative Commons Attribution Non-Commercia License (https://creativecommons.org/licenses/by-nc/4.0/) which permits unrestricted non-commercial use, distribution, and reproduction in any medium, provided the original work is properly cited.

C) 2021 Korean Wound Management Society 
was necessary after palliative mastectomy [5]. However, these studies did not directly compare the surgical results between patients requiring a reconstructive operation and those who did not. In addition, studies on reconstructive surgery usually only included the split-thickness skin graft (STSG) method.

Therefore, we evaluated the outcomes of various reconstructive methods after mastectomy for palliative purposes in patients with stage-IV breast cancer at a single institution over the past 10 years. We aimed to determine the postoperative satisfaction of patients who underwent reconstruction after palliative mastectomy and to determine whether additional reconstruction methods affect the survival rates after palliative mastectomy.

\section{Methods}

\section{Patients}

Between April 2010 and April 2021, 102 patients underwent palliative mastectomy, of which 62 and 40 underwent primary closure and reconstruction, respectively. Data of patients who underwent reconstructive surgery after palliative mastectomy (i.e., patients' age, sex, diagnosis, stage, surgical method, excised skin area, reconstruction method, complications, metastasis site, follow-up period, and survival time) were collected and reviewed retrospectively. The study was approved by the Institutional Review Board of Seoul National University Hospital (IRB No. H-2108-081-1245) and performed in accordance with the principles of the Declaration of Helsinki. The patients provided written informed consent for the publication and the use of their images.

\section{Operation}

In the preoperative stage, patients with potential for reconstructive surgery after palliative mastectomy were selected, and the plastic surgery team was prepared for reconstructive surgery. After the general surgeons performed palliative mastectomy, thoracic surgeons performed chest wall resection when the tumor had invaded the chest wall. Whether or not reconstructive surgery was actually performed was decided depending on whether primary closure by general surgeons was possible after palliative mastectomy, or whether the patient wanted to undergo cosmetic reconstructive surgery. Plastic surgeons performed reconstruction based on the following: location, size, and depth of the defect; damaged structures; and past surgical history. In many reconstruction cases, STSG was considered first; however, when it was determined that recon- struction with skin graft alone was impossible because of an extensive resection, flap surgery was planned preoperatively.

\section{Evaluation}

To determine the effect of palliative total mastectomy and subsequent reconstruction on the patient's overall health and quality of life, we surveyed the change in these indicators before and after surgery through questionnaires. Patient satisfaction after surgery was investigated using the Quality of Life Questionnaire Core 30-questions, and Quality of Life Questionnaire-Breast Cancer-23-questions of the European Organisation for Research and Treatment of Cancer. Afterwards, survival analysis was performed to determine if there were any differences in the survival rates depending on the reconstruction method. Survival was censored at the time of the last follow-up examination, and the unadjusted overall survival was estimated using the Kaplan-Meier survival analysis [6].

\section{Statistical analysis}

Patient data were analyzed using univariate methods. Age, skin resection area, postoperative hospital stay, and postoperative survival period were compared between the reconstruction and primary closure groups through an independent sample $\mathrm{t}$-test. Then, the same analysis was performed between the skin graft and the flap surgery groups within the reconstruction group. The results are presented as means \pm standard deviations. Statistical analyses were performed using PASW Statistics 18 (IBM Corp., Armonk, NY, USA). Statistical significance was set at $\mathrm{P}<0.05$.

\section{Results}

Patient demographics, reconstruction methods, and postoperative results are summarized in Table 1. The mean age of the patients at the time of reconstruction was 50.3 years (range, 23-71 years). The average period from diagnosis to reconstruction was 15.1 months (range, 3-81 months). Reconstruction methods included free transverse rectus abdominis musculocutaneous (TRAM) flap $(n=3)$, pedicled latissimus dorsi (LD) flap $(n=3)$, pedicled TRAM flap $(n=2)$, pedicled LD with pedicled vertical rectus abdominis musculocutaneous (VRAM) flap $(n=1)$, tissue expander insertion $(n=3)$, and STSG $(n=28)$. STSG comprised $70 \%$ of all reconstructions, with a mean area of $253 \mathrm{~cm}^{2}$. There were no significant complications due to reconstructive surgery. After STSG, there was one case of partial (>30\%) graft loss, one case of breast sero- 
Table 1. Patient demographics in reconstruction group

\begin{tabular}{lc}
\hline Characteristics & No. of patients $(\mathrm{n}=40)$ \\
\hline Mean age at operation $(\mathrm{yr})$ & 50.3 \\
Mean excised skin $\left(\mathrm{cm}^{2}\right)$ & 253 \\
Reconstruction method & \\
STSG & 28 \\
Free TRAM flap & 3 \\
Pedicled LD flap & 3 \\
Pedicled TRAM flap & 2 \\
Pedicled LD and VRAM flap & 1 \\
TEl & 3 \\
Complications & 5 \\
Partial skin graft loss & 1 \\
Seroma & 2 \\
Pneumonia & 1 \\
Empyema & 1
\end{tabular}

STSG, split-thickness skin graft; TRAM, transverse rectus abdominis musculocutaneous; LD, latissimus dorsi; VRAM, vertical rectus abdominis musculocutaneous; TEl, tissue expander insertion. ma, one case of axillary seroma, one case of pneumonia, and one case of empyema. However, there was no case of flap failure. All lost skin underwent secondary healing, and there were no cases of reconstruction-related revision surgery.

The patient data for the reconstruction group are summarized in Table 2. On pathological examination, 67.5\% (27/40) of the reconstruction patients had invasive ductal carcinoma, followed by invasive lobular carcinoma, inflammatory breast cancer, and malignant phyllodes tumor in decreasing order. The average hospital stay after reconstruction was 8.68 days. The patients started conventional chemotherapy and radiotherapy at an average of 31.3 and 91.5 days after undergoing reconstructive surgery, respectively. After completion of softtissue healing, the global health score as assessed by the questionnaire improved from $51 \%$ preoperatively to $78 \%$ postoperatively. In addition, the quality of life reported by the patients was comparable to or slightly improved with global health, from $37 \%$ before surgery to $83 \%$ postoperatively.

The mean survival time of patients after reconstructive surgery was 43.9 months (range, 1-129 months). There was sig-

Table 2. Patient data in reconstruction group

\begin{tabular}{|c|c|c|c|c|c|c|c|c|c|c|c|c|c|}
\hline №. & $\begin{array}{c}\text { Sex/ } \\
\text { age (yr) }\end{array}$ & $\begin{array}{c}\text { GS } \\
\text { operation }\end{array}$ & $\begin{array}{l}\text { Excised skin } \\
\quad(\mathrm{cm})\end{array}$ & Reconstruction & Complication & $\begin{array}{l}\text { HS } \\
\text { (day) }\end{array}$ & Diagnosis & Stage & Metastasis & $\begin{array}{c}\text { Survival } \\
\text { time (mo) }\end{array}$ & NeoTx & CTx & RTx \\
\hline 1 & $\mathrm{~F} / 50$ & TM with ALND & $17 \times 12$ & Pedicled LD, STSG & - & 9 & IDC & T3N2M1 & Skin, muscle & $>129$ & + & + & + \\
\hline 2 & $\mathrm{~F} / 40$ & TM with ALND & $6.2 \times 5.8$ & Free TRAM & - & 7 & IDC & T3N+M1 & Bone & 61 & + & + & + \\
\hline 3 & $F / 38$ & WE & $17.2 \times 5.6$ & STSG & - & 13 & IDC & T4N3M1 & Lung, liver & 2 & + & + & + \\
\hline 4 & $\mathrm{~F} / 57$ & TM with ALND & $16 \times 15$ & STSG & - & 12 & IDC & T4N3M1 & SCN, peritoneum & 5 & + & + & + \\
\hline 5 & $\mathrm{~F} / 51$ & TM with ALND & $13.5 \times 8.8$ & STSG & Pneumonia & 6 & $\mathrm{MBC}$ & T3N1M1 & Bone, lung & 14 & + & + & + \\
\hline 6 & $F / 57$ & SSM with ALND & $10.5 \times 4.6$ & TEl & Seroma & 6 & IDC & T2NxM1 & Bone, liver & 11 & + & + & + \\
\hline 7 & $\mathrm{~F} / 48$ & TM with ALND & $26.5 \times 15$ & Free TRAM & - & 11 & IDC & T4N3M1 & Lung, brain & 50 & + & + & + \\
\hline 8 & $F / 43$ & TM with ALND & $15.0 \times 11.0$ & STSG & - & 12 & IDC & T4NOM1 & Brain & 4 & + & + & + \\
\hline 9 & $F / 46$ & TM with ALND & $9.0 \times 4.5$ & Pedicled LD & - & 5 & ILC & T3N3M1 & Liver, lung, bone & $>47$ & + & + & + \\
\hline 10 & $\mathrm{~F} / 48$ & SSM with ALND & $10.0 \times 3.5$ & TEl & - & 5 & IDC & T2N1M1 & Bone, liver & $>43$ & + & + & + \\
\hline 11 & $\mathrm{~F} / 53$ & TM with ALND & $18.0 \times 19.0$ & STSG & - & 6 & IDC & T4N3M1 & Lung & $>15^{\mathrm{a})}$ & + & + & - \\
\hline 12 & $F / 36$ & TM with ALND & $14.0 \times 12.0$ & STSG & - & 8 & IDC & T4N3M1 & Skin & $>37$ & + & + & - \\
\hline 13 & $F / 29$ & TM with ALND & $19.5 \times 17.5$ & Free TRAM & Seroma & 11 & IDC & T4N3M1 & Lung, liver & 12 & + & + & + \\
\hline 14 & $\mathrm{~F} / 53$ & TM with ALND & $17.0 \times 16.0$ & STSG & - & 7 & IDC & T4N3M1 & Lung & $>5^{a)}$ & + & + & + \\
\hline 15 & $\mathrm{~F} / 42$ & TM with ALND & $16.7 \times 12.0$ & STSG & - & 8 & $\mathrm{IBC}$ & T4N3M1 & Brain, liver, lung & 14 & + & + & + \\
\hline 16 & $\mathrm{~F} / 71$ & TM with ALND & $17.0 \times 16.1$ & STSG & - & 8 & PLC & T3NOM1 & Bone, lung & 8 & + & + & + \\
\hline 17 & $\mathrm{~F} / 28$ & TM with ALND & $16.5 \times 12.5$ & STSG & - & 8 & ILC & T3N3M1 & Liver, brain & 5 & + & + & + \\
\hline 18 & $\mathrm{~F} / 50$ & TM with ALND & $15.5 \times 13.0$ & STSG & - & 6 & IDC & T3N1M1 & Skin & $>26$ & + & + & + \\
\hline 19 & $\mathrm{~F} / 50$ & TM with ALND & $20.0 \times 13.0$ & STSG & - & 6 & IDC & T3N3M1 & Lung, liver & 9 & + & + & + \\
\hline 20 & $F / 54$ & $\begin{array}{l}\text { Chest wall } \\
\text { resection }\end{array}$ & $10.0 \times 11.0$ & Pedicled TRAM & - & 8 & NA & TxN3M1 & Lung & $>27$ & + & + & + \\
\hline
\end{tabular}


Yoon T et al.

Reconstruction after palliative mastectomy

Table 2. Continued

\begin{tabular}{|c|c|c|c|c|c|c|c|c|c|c|c|c|c|}
\hline №. & $\begin{array}{c}\text { Sex/ } \\
\text { age (yr) }\end{array}$ & $\begin{array}{c}\text { GS } \\
\text { operation }\end{array}$ & $\begin{array}{l}\text { Excised skin } \\
\quad(\mathrm{cm})\end{array}$ & Reconstruction & Complication & $\begin{array}{c}\text { HS } \\
\text { (day) }\end{array}$ & Diagnosis & Stage & Metastasis & $\begin{array}{l}\text { Survival } \\
\text { time (mo) }\end{array}$ & NeoTx & CTx & RTx \\
\hline 21 & $F / 57$ & TM with ALND & $16.5 \times 14.0$ & STSG & - & 10 & IDC & T3N2M1 & Liver & $>26$ & + & + & + \\
\hline 22 & $F / 39$ & TM with ALND & $18.0 \times 13.0$ & STSG & - & 7 & IDC & T4N3M1 & Brain, lymph node & $>11^{\mathrm{a})}$ & + & + & + \\
\hline 23 & $\mathrm{~F} / 72$ & TM with ALND & $24.5 \times 18.5$ & STSG & - & 9 & IDC & T4N3M1 & Liver & 2 & + & + & + \\
\hline 24 & $F / 69$ & TM with ALND & $21.0 \times 17.5$ & STSG & - & 7 & IDC & T4N2M1 & Skin & $>24$ & + & + & + \\
\hline 25 & $F / 63$ & $\mathrm{TM}$ & $15.5 \times 7.5$ & STSG & - & 6 & IMC & T4N3M1 & Brain, breast & $>24$ & + & + & + \\
\hline 26 & $\mathrm{~F} / 57$ & WE & $15.0 \times 4.0$ & Pedicled LD & - & 6 & Angiosarcoma & T2NxM1 & Lung & $>13^{\mathrm{a})}$ & + & + & + \\
\hline 27 & $\mathrm{~F} / 50$ & TM with ALND & $18.0 \times 14.0$ & STSG & - & 8 & IDC & T3N1M1 & Skin & $>20$ & + & + & + \\
\hline 28 & $F / 44$ & TM with ALND & $17.5 \times 14.5$ & STSG & - & 8 & IDC & T4N2M1 & Lymph node & $>19$ & + & + & + \\
\hline 29 & $F / 44$ & TM with ALND & $16.0 \times 11.5$ & STSG & - & 7 & IDC & T4N2M1 & Lung, liver & 12 & + & + & + \\
\hline 30 & $F / 44$ & WE with ALND & $30.0 \times 25.0$ & Pedicled LD, VRAM & Empyema & 27 & IMPC & T3N2M1 & Skin & 9 & + & + & + \\
\hline 31 & $F / 55$ & TM with SLNB & $20.5 \times 14.0$ & STSG & - & 7 & IDC & T4N1M1 & Bone, skin & $>7^{\text {a) }}$ & + & + & + \\
\hline 32 & $F / 52$ & TM with ALND & $20.5 \times 15.0$ & STSG & - & 10 & MPNST & Grade 3 & Lung, LN, thigh & 11 & + & + & + \\
\hline 33 & $\mathrm{~F} / 71$ & $\mathrm{TM}$ & $20.0 \times 14.0$ & STSG & - & 9 & MPT & Malignant & Lung & 3 & + & + & - \\
\hline 34 & $F / 35$ & TM with ALND & $29.0 \times 23.5$ & STSG & Partial graft loss & 12 & IDC & T4NOM1 & Skin, lung, liver & 5 & + & + & - \\
\hline 35 & $\mathrm{~F} / 70$ & TM with ALND & $15.0 \times 6.0$ & STSG & - & 10 & IDC & T4NOM1 & Breast, lymph node & $>7^{\text {a) }}$ & + & + & + \\
\hline 36 & $F / 23$ & SSM with SLNB & $7.7 \times 4.3$ & TEl & - & 5 & IDC & T4N2M1 & Bone & $>11$ & + & + & + \\
\hline 37 & $\mathrm{~F} / 62$ & TM with ALND & $20.8 \times 13.5$ & STSG & - & 5 & IDC & T3N3M1 & Bone & $>9$ & + & + & + \\
\hline 38 & $F / 61$ & TM with ALND & $22.0 \times 19.0$ & STSG & - & 14 & MPT & Malignant & Lung, bone & 2 & + & - & + \\
\hline 39 & $F / 43$ & TM with ALND & $18.0 \times 15.0$ & Pedicled TRAM & - & 8 & IDC & T4N1M1 & Skin, lymph node & $>8$ & + & + & + \\
\hline 40 & $\mathrm{~F} / 60$ & TM with ALND & $39.0 \times 21.0$ & STSG & - & 10 & $\mathrm{IBC}$ & T4N3M1 & Skin & $>4$ & + & + & - \\
\hline
\end{tabular}

GS, general surgery; HS, hospital stay after reconstruction; NTx, neoadjuvant therapy; CTx, chemotherapy; RTx, radiation therapy; F, female; M, male; TM, total mastectomy; ALND, axillary lymph node dissection; WE, wide excision; SSM, skin-sparing mastectomy; SLNB, sentinel lymph node biopsy; LD, latissimus dorsi; STSG, split-thickness skin graft; TRAM, transverse rectus abdominis musculocutaneous; VRAM, vertical rectus abdominis musculocutaneous; TEI, tissue expander insertion; IDC, invasive ductal carcinoma; MBC, metaplastic breast cancer; IBC, inflammatory breast cancer; PLC, pleomorphic lobular carcinoma; ILC, invasive lobular carcinoma; IMC, invasive mammary carcinoma; IMPC, invasive micropapillary carcinoma; MPNST, malignant peripheral nerve sheath tumor; MPT, malignant phyllodes tumor; SCN, supraclavicular lymphnode.

a)Lost to follow-up.

nificant difference in the survival rate between the patients who underwent reconstruction and those who underwent primary closure after palliative mastectomy $(\mathrm{P}<0.05)($ Fig. $1 \mathrm{~A})$. There was no significant difference in the survival rate between the patients who underwent reconstruction with STSG and those who underwent reconstruction with flap $(\mathrm{P}>0.05)$ (Fig. 1B). Group analysis and reconstruction subgroup analysis results are summarized in Tables 3 and 4, respectively.

The cases of patients \#7 and \#30 are representative cases of patients who underwent reconstruction.

Patient \#7 was a 52-year-old woman who was diagnosed with invasive ductal carcinoma of the left breast, stage cT4N3M1. Palliative mastectomy with chest wall resection and en bloc resection of the third and fourth ribs were performed. The defect was $30 \times 15 \mathrm{~cm}$ in size with exposure of the GoreTex mesh after chest wall resection. Elevation of a $30 \times 14-\mathrm{cm}$ sized free TRAM flap was performed to resurface the chest wall. Anastomosis was performed with the internal mammary artery in an end-to-end manner. There were no complications, and the patient received postoperative adjuvant chemotherapy and radiotherapy. At 50 months postoperatively, the patient expired due to metastases to the brain and lungs (Fig. 2).

Patient \#30 was a 44-year-old woman who was diagnosed with invasive micropapillary carcinoma of the right breast, stage cT3N2M1 with PIK3CA, M1043V, and NTRK1 mutations. Palliative mastectomy and chest wall resection were performed, leaving a $35 \times 15-\mathrm{cm}$ defect exposing the pericardium. First, a $15 \times 15-\mathrm{cm}$ Gore-Tex dual mesh was applied to cover the pericardium. Next, pedicled LD and pedicled VRAM flaps from the contralateral side were elevated to cover the defect. After insetting of the flaps and approximation of the surrounding tissue, STSG was applied to the remaining defect. 

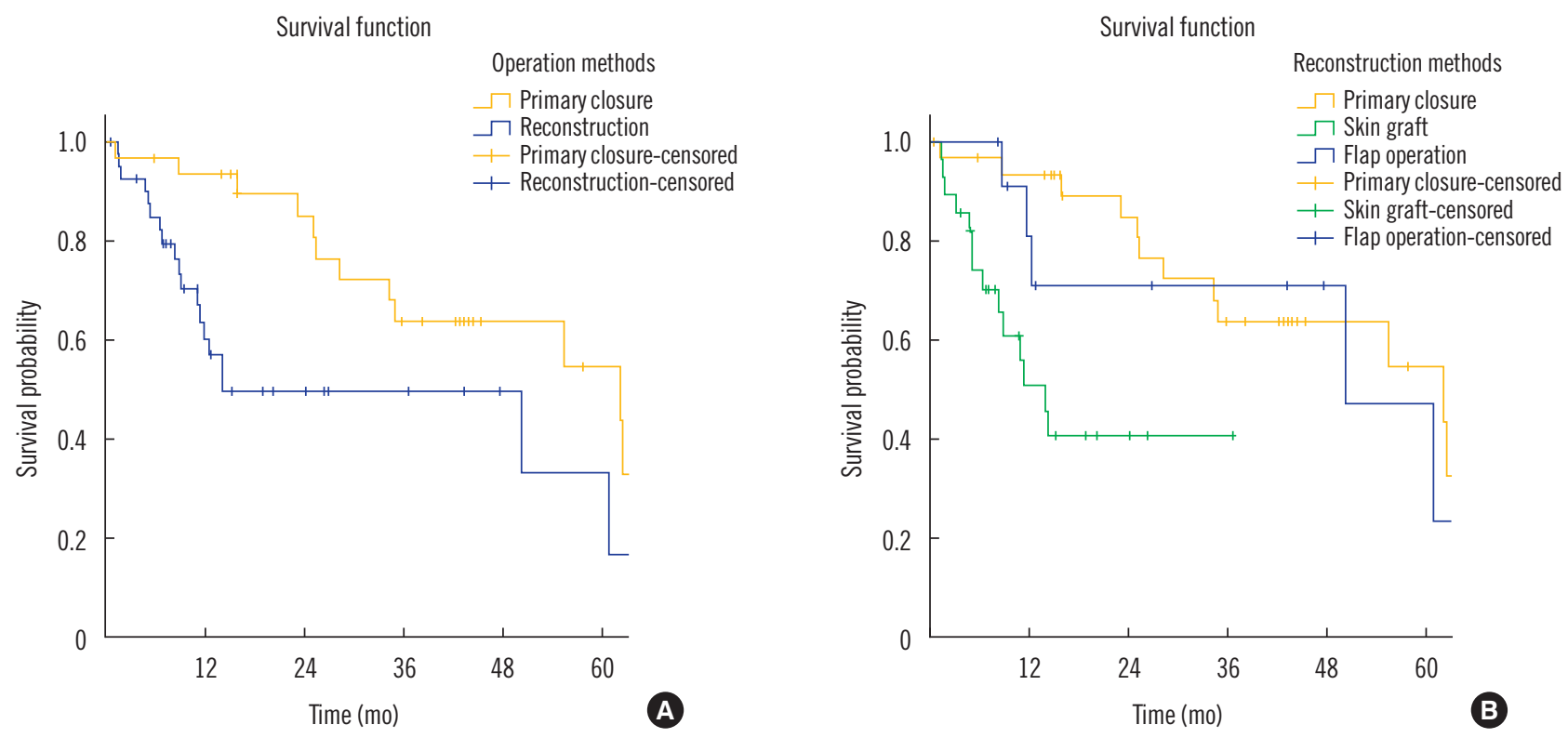

Fig. 1. Kaplan-Meier curve for overall survival $(n=102)$. Survival probability of patients that underwent palliative mastectomy. (A) There was a significant difference in the survival probability between the patients who underwent reconstruction and those who underwent primary closure after palliative mastectomy $(\mathrm{P}<0.05)$. (B) There was no significant difference in the survival probability between the patients who underwent reconstruction using split-thickness skin graft and those who underwent reconstruction using various flaps $(P>0.05)$.

Table 3. Group analysis

\begin{tabular}{lccc}
\hline Operation & $\begin{array}{c}\text { Reconstruction } \\
\text { group }(\mathrm{n}=40)\end{array}$ & $\begin{array}{c}\text { Primary closure } \\
\text { group }(\mathrm{n}=62)\end{array}$ & P-value \\
\hline Mean age (yr) & 50.3 & 49.9 & 0.87 \\
Mean excised skin $\left(\mathrm{cm}^{2}\right)$ & 253 & 147 & 0.01 \\
Mean hospital stay (day) & 8.7 & 4.8 & $<0.001$ \\
Survival time (mo), median \pm SD & $14.0 \pm 14.2$ & $62.3 \pm 18.6$ & 0.03 \\
\hline
\end{tabular}

Although the patient received palliative chemotherapy and radiotherapy, metastases to the left breast and lymph nodes occurred. She survived for 8 months after surgery (Fig. 3).

\section{Discussion}

Palliative mastectomy is performed for symptomatic and local control in patients with advanced breast cancer with metastatic lesions [1]. Palliative mastectomy can relieve patients' symptoms, optimize local control, improve the overall health and quality of life, and even increase life expectancy [7]. The efficacy of palliative mastectomy in patients with advanced breast cancer remains controversial [7-10]. While there are several reports stating that palliative mastectomy does not improve the survival rate $[9,11]$, many studies have also shown that the survival rate can be improved by resection of the primary tu-
Table 4. Reconstruction subgroup analysis

\begin{tabular}{lccc}
\hline Reconstruction & $\begin{array}{c}\text { STSG group } \\
(\mathrm{n}=28)\end{array}$ & $\begin{array}{c}\text { Flap group } \\
(\mathrm{n}=9)\end{array}$ & P-value \\
\hline Mean age $(\mathrm{yr})$ & 52.6 & 45.7 & 0.11 \\
Mean excised skin $\left(\mathrm{cm}^{2}\right)$ & 278 & 245 & 0.63 \\
Mean hospital stay (day) & 8.5 & 10.2 & 0.25 \\
Survival time (mo), median \pm SD & $14.0 \pm 2.1$ & $50.2 \pm 22.1$ & 0.07 \\
\hline
\end{tabular}

STSG, split-thickness skin graft.

mor along with an increase in the metastasis-free survival period $[12,13]$. Large-scale meta-analyses have confirmed that the survival rate could be increased by locoregional therapy, which involves localized radiotherapy after primary tumor resection $[14,15]$. Because cancer induces an immunosuppressive state, by removing the source, partial restoration of immunity can be expected [16]. It is hypothesized that palliative mastectomy may reduce the tumor burden and increase life expectancy by eliminating the source of metastasis [17]. Therefore, we focused on reconstructive surgery after palliative mastectomy.

Reconstructive surgery is performed by plastic surgeons when primary closure is not possible because of a large skin defect after mastectomy, or when muscle resection or chest wall resection has been performed. Aided by skin laxity, primary closure is performed in more than half of the patients af- 
Yoon T et al.

Reconstruction after palliative mastectomy
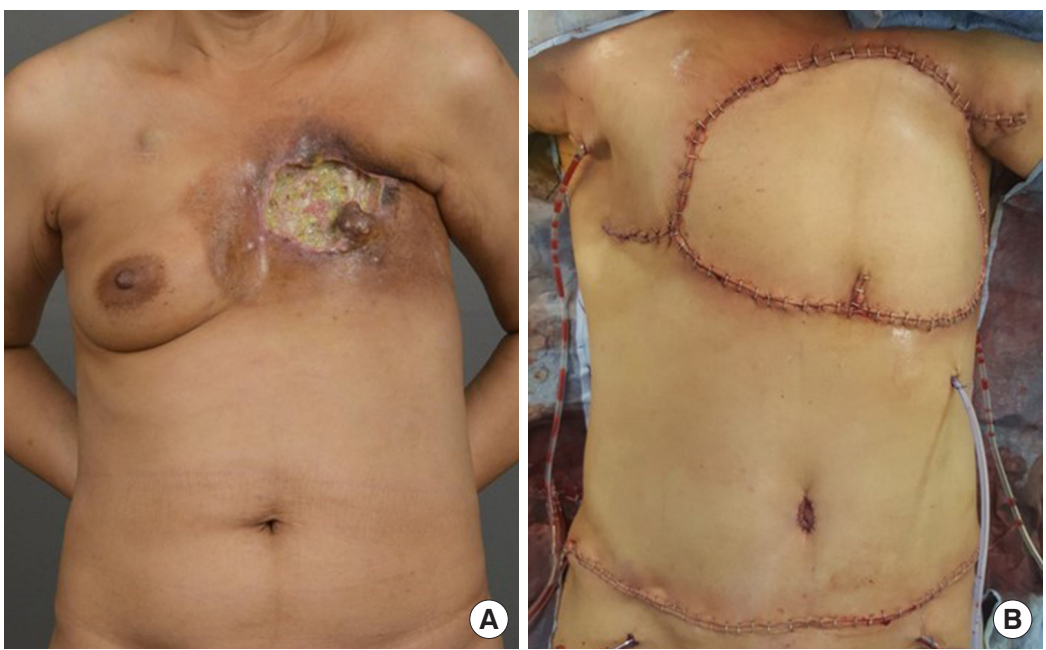

Fig. 2. Reconstruction using free transverse rectus abdominis musculocutaneous (TRAM) flap. (A) Stage cT4N3M0 invasive ductal carcinoma of the left breast. Palliative mastectomy with chest wall resection along with en bloc resection of the third and fourth ribs was performed. The defect was $30 \times 15 \mathrm{~cm}$ in size. (B) After chest wall resection, a $30 \times 14-\mathrm{cm}$ sized free TRAM flap was elevated and inset to resurface the chest wall.
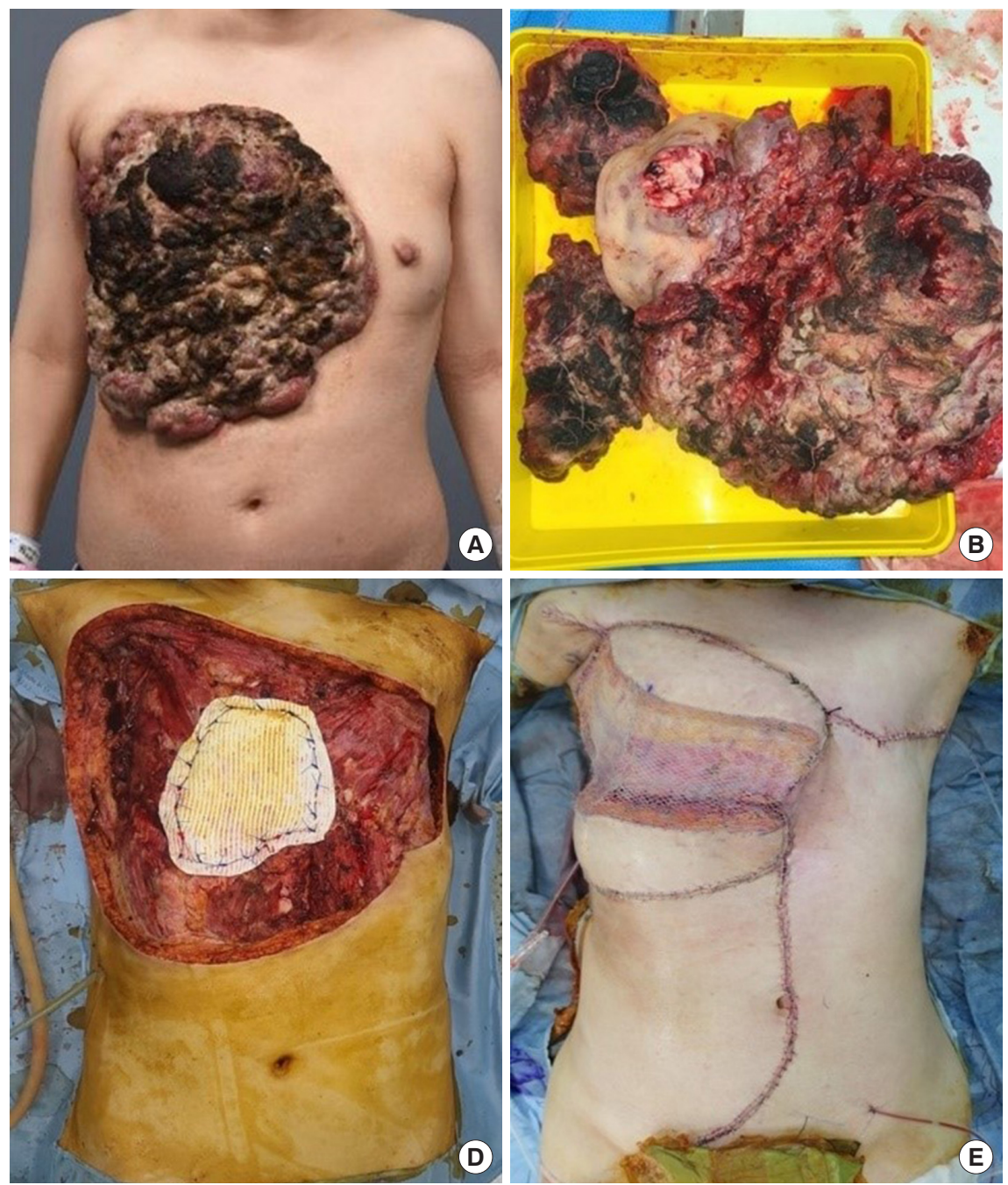

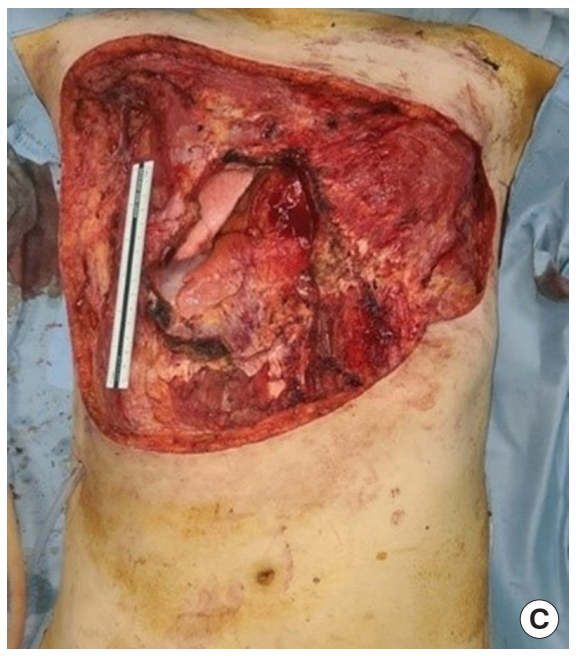

Fig. 3. Reconstruction using pedicled LD flap, pedicled VRAM flap, and STSG. (A) Before surgery, a $30 \times 29-\mathrm{cm}$ mass invaded the entire right breast and part of the left breast. (B) The right breast $(30 \times 25 \times 7.5$ $\mathrm{cm})$, chest wall $(11.5 \times 10 \times 1 \mathrm{~cm})$, and left breast $(16 \times 12.5 \mathrm{~cm})$ were excised through a wide excision. (C) Reconstruction of the extensive defects was planned using pedicled LD and pedicled VRAM flaps. (D) The chest wall was reconstructed using a Gore-Tex mesh. (E) Photographs were taken immediately after surgery. LD, latissimus dorsi; VRAM, vertical rectus abdominis musculocutaneous; STSG, splitthickness skin graft. 
ter mastectomy for stage-IV lesions in our hospital. Based on our data, even in patients with stage-IV breast cancer, reconstruction was completed only with skin grafts and local flaps in approximately $70 \%$ of the cases. Even if reconstruction was performed using a free flap or a pedicled flap, there were only cases of general postoperative discomfort after general anesthesia, such as pain, nausea, vomiting, sore throat, and thirst. Even after performing a relatively large surgery involving free TRAM flap or pedicled LD and pedicled VRAM flaps, there were no major complications due to reconstruction. According to the results of this study, palliative mastectomy can be performed regardless of the complexity of reconstruction because plastic reconstruction does not cause major complications affecting the patient's subsequent treatment. However, successful palliative mastectomy requires a well-prepared team of plastic surgeons.

There are insufficient studies on reconstruction after palliative mastectomy. To our knowledge, there is no study to date on the difference in the survival rate according to the performed reconstruction method after palliative mastectomy. In this study, there was a difference in the survival rate of the reconstruction group compared to the group in which primary closure was possible. The skin defect area was significantly smaller in the cases where primary closure was possible without reconstruction after palliative mastectomy, and the survival rate was significantly higher. Although not included in the analysis, among the patients who underwent mastectomy for curative purposes for stage-III locally advanced breast cancer and those who underwent reconstruction including STSG due to the large size of the lesion, there were approximately 10 cases of stage-IV metastasis during the follow-up period. These results were consistent with those of previous studies showing that a larger tumor acts as a factor that reduces survival in breast cancer [14]. Nevertheless, when reconstruction of extensive soft-tissue defects was performed after palliative mastectomy, an increase in mortality due to reconstruction itself was not confirmed.

Various reconstruction methods have been established for defects occurring after mastectomy [18]. Breast reconstruction using pedicled flaps has been practiced since the early 20th century, and reconstruction using free flaps has also been implemented since the late 20th century. It is currently known that immediate reconstruction after mastectomy does not increase the risk of cancer recurrence and has no effect on survival [19]. However, there are few studies on immediate reconstruction after palliative mastectomy. In this study, in patients who underwent reconstruction after palliative mastectomy, the survival rate was higher in flap reconstruction than in STSG cases $(P=0.079)$. This result was of great importance considering that flap-based reconstruction was usually performed when the tumor infiltrated the surrounding muscle or bone and could not be reconstructed through skin grafting. In cases of flap surgery, there is a possibility that chemotherapy and radiation therapy were more effective, thus, increasing the survival rate. As the number of samples was small and no statistically significant results were obtained, it may be difficult to state that flap surgery improves the survival rate after palliative mastectomy compared to skin grafting. Although flap surgery is a more complex reconstruction method than skin grafting, it is meaningful because the survival rate as well as complications were not high. This could be a more viable option for younger patients who can tolerate flap surgery better. Further research is needed in this regard.

In addition to the survival gain, palliative mastectomy is necessary to improve the quality of life. Palliative mastectomy should be performed to maximally control the symptoms, prevent serious complications, and prolong life while minimizing impairment of lifestyle and quality of life [20]. Although the questionnaire investigating quality of life before and after surgery was administered only to a limited number of patients, mastectomy and subsequent reconstruction were found to significantly improve the quality of life in our study. Admittedly, this included the effect of total mastectomy itself, but it is impossible to improve quality of life without performing proper reconstruction. Although we focused on unavoidable reconstruction after mastectomy, some patients may wish to undergo reconstruction for better quality of life during survival. In stage-IV patients who respond well to systemic therapy and who are expected to survive longer than average, cosmetic breast reconstruction may also be considered [21]. Reconstruction with a TRAM flap was performed to cover extensive soft-tissue defects, and a favorable outcome was confirmed postoperatively. Reconstruction using autologous tissue to replace skin grafts may also be attempted, and can improve the quality of life. When performing cosmetic reconstruction for stage-IV breast cancer patients, special attention should be paid to the indications. Continuing efforts are needed to improve the quality of life of patients with advanced breast cancer after palliative mastectomy by performing appropriate reconstruction surgery after considering the cosmetic aspects.

This study had several limitations. Although a patient-con- 
trolled study was ideal to confirm the effects of reconstruction after mastectomy, it was difficult to distinguish and analyze patients who did not undergo palliative mastectomy despite requiring palliative mastectomy. In addition, there were cases where further follow-up examination was not possible because the patient did not survive for a long time. Therefore, it was not possible to obtain a complete dataset for all elements to be comparatively analyzed. Questionnaires on global health and quality of life were collected only from patients who underwent reconstruction, excluding those who underwent only primary closure after palliative mastectomy. In addition, as the prognosis after palliative mastectomy and reconstruction can be greatly affected by the hormone receptors, a subgroup analysis by receptor type should have been performed, but this was not possible because the number of participants was not large enough. Despite these limitations, this study is valuable as it provides demographic data on the postoperative outcomes of palliative mastectomy and reconstruction performed in patients with stage-IV breast cancer.

In conclusion, this study reported the reconstruction outcomes after performing palliative mastectomy using skin grafts and various flaps in patients with advanced breast cancer. Reconstruction methods immediately after palliative mastectomy did not affect patient survival. Successful reconstruction after mastectomy enabled a faster return and improved quality of life as well as a chance to receive further treatment. The complexity of reconstructive surgery of the soft-tissue defect that occurs after palliative mastectomy should not be a major consideration when determining the indications of palliative surgery.

\section{Conflict of interest}

No potential conflict of interest relevant to this article was reported.

\section{ORCID iDs}

Taekeun Yoon https://orcid.org/0000-0002-1070-5165

Soo Jin Woo https://orcid.org/0000-0003-3476-8133

Ung Sik Jin

https://orcid.org/0000-0001-5096-6207

\section{References}

1. Morrogh M, Park A, Norton L, et al. Changing indications for surgery in patients with stage IV breast cancer: a cur- rent perspective. Cancer 2008;112:1445-54.

2. Blanchard DK, Shetty PB, Hilsenbeck SG, et al. Association of surgery with improved survival in stage IV breast cancer patients. Ann Surg 2008;247:732-8.

3. Chung CT, Carlson RW. Goals and objectives in the management of metastatic breast cancer. Oncologist 2003;8: 514-20.

4. Vieira RA, da Silva KM, de Oliveira-Junior I, et al. ITADE flap after mastectomy for locally advanced breast cancer: a good choice for mid-sized defects of the chest wall, based on a systematic review of thoracoabdominal flaps. J Surg Oncol 2017;115:949-58.

5. Durrant CA, Khatib M, Macneill F, et al. Mastectomy and reconstruction in stage IV breast cancer: a survey of UK breast and plastic surgeons. Breast 2011;20:373-9.

6. Kaplan EL, Meier P. Nonparametric estimation from incomplete observations. J Am Stat Assoc 1958;53:457-81.

7. Lane WO, Thomas SM, Blitzblau RC, et al. Surgical resection of the primary tumor in women with de novo stage IV breast cancer: contemporary practice patterns and survival analysis. Ann Surg 2019;269:537-44.

8. Harris E, Barry M, Kell MR. Meta-analysis to determine if surgical resection of the primary tumour in the setting of stage IV breast cancer impacts on survival. Ann Surg Oncol 2013;20:2828-34.

9. Rosche M, Regierer AC, Schwarzlose-Schwarck S, et al. Primary tumor excision in stage IV breast cancer at diagnosis without influence on survival: a retrospective analysis and review of the literature. Onkologie 2011;34:607-12.

10. Soran A, Ozmen V, Ozbas S, et al. Randomized trial comparing resection of primary tumor with no surgery in stage IV breast cancer at presentation: protocol MF07-01. Ann Surg Oncol 2018;25:3141-9.

11. Dominici L, Najita J, Hughes M, et al. Surgery of the primary tumor does not improve survival in stage IV breast cancer. Breast Cancer Res Treat 2011;129:459-65.

12. Bafford AC, Burstein HJ, Barkley CR, et al. Breast surgery in stage IV breast cancer: impact of staging and patient selection on overall survival. Breast Cancer Res Treat 2009; 115:7-12

13. Babiera GV, Rao R, Feng L, et al. Effect of primary tumor extirpation in breast cancer patients who present with stage IV disease and an intact primary tumor. Ann Surg Oncol 2006; 13:776-82.

14. Carter CL, Allen C, Henson DE. Relation of tumor size, lymph node status, and survival in 24,740 breast cancer 
cases. Cancer 1989;63:181-7.

15. Gera R, Chehade HE, Wazir U, et al. Locoregional therapy of the primary tumour in de novo stage IV breast cancer in 216,066 patients: a meta-analysis. Sci Rep 2020;10:2952.

16. Danna EA, Sinha P, Gilbert M, et al. Surgical removal of primary tumor reverses tumor-induced immunosuppression despite the presence of metastatic disease. Cancer Res 2004;64:2205-11.

17. Rashid OM, Nagahashi M, Ramachandran S, et al. Resection of the primary tumor improves survival in metastatic breast cancer by reducing overall tumor burden. Surgery
2013;153:771-8.

18. Uroskie TW, Colen LB. History of breast reconstruction. Semin Plast Surg 2004;18:65-9.

19. Nedumpara T, Jonker L, Williams MR. Impact of immediate breast reconstruction on breast cancer recurrence and survival. Breast 2011;20:437-43.

20. Hortobagyi GN. Treatment of breast cancer. N Engl J Med 1998;339:974-84.

21. Asaad M, Meaike J, Yonkus J, et al. Breast reconstruction in the setting of stage 4 breast cancer: is it worthwhile? Ann Surg Oncol 2020;27:4730-9. 